\title{
Synthesis and Characterization of Un-encapsulated and Pterostilbene-encapsulated DOTAP: Cholesterol Liposomes
}

\author{
Kaustubh Sunil Hiray, Suresh Palamadai Krishnan* \\ Department of Biomedical Sciences, School of Biosciences and Technology, VIT, Vellore, Tamil Nadu, INDIA.
}

\begin{abstract}
Aim: Our study focuses on the liposome-based nanoformulation, which can encapsulate Pterostilbene for its subsequent testing in relevant, model systems for cancer. Background: Pterostilbene, a plant-derived, hydrophobic, dietary stilbenoid, has been studied for its ability to induce cell death and regulate caspases in the different types of cancer cells. The potential of this drug can be improved by formulating a suitable vehicle for its delivery. Biocompatible, lipid-based nanoparticles called liposomes have been studied as a potent delivery vehicle for drugs in pre-clinical as well as in clinical studies. Liposomes can improve the drug uptake and bioavailability of the drugs. Materials and Methods: Pterostilbene loaded liposomes were constructed using DOTAP and Cholesterol, by the Thin-Film Hydration method. Along with the loaded liposomes, blank liposomes (only DOTAP and Cholesterol, without Pterostilbene) were also constructed. The liposomes were characterized for their size, Polydispersity Index (PDI) and Zeta potential using DLS. Shape of the liposomes was analysed using TEM. Encapsulation Efficiency (EE) of the Pterostilbene loaded liposomes was determined. Also, UV-Vis spectrophotometer was used to ensure that Pterostilbene was encapsulated inside the liposomes and there was no interaction between the drug and the lipids. Results: Liposomes were composed of DOTAP and Cholesterol with molar ratios 2:1. The DLS showed that the size of the Pterostilbene-loaded liposomes was $435.6 \pm 5 \mathrm{~nm}(n=3)$, PDI was $0.5 \pm 0.07(n=3)$ and Zeta potential was $-16.4 \pm 0.5 \mathrm{mV}(n=3)$. The drug encapsulation efficiency was found to be $97.5 \pm 0.8 \%(n=3)$. Conclusion: Reproducibility in the results (DLS and EE data for Pterostilbene-encapsulated liposomes) provides a sound, scientific basis for evaluating their cell death potential of Pterostilbene loaded liposomes against cancer cells in comparison with that of free Pterostilbene (parent compound). Also, the experimental flow of ours can be used as a teaching tool by educators in drug delivery and allied fields. Key words: Pterostilbene, DOTAP, Cholesterol, Liposomes, Nanoparticles.
\end{abstract}

\section{INTRODUCTION}

Pterostilbene (IUPAC name- 4-[(E)-2-(3,5dimethoxyphenyl)ethenyl]phenol) is a naturally occurring, hydrophobic compound and its relatively better cell membrane permeability has been attributed to be mainly due to it having two dimethoxy groups. It is mostly found in grapes, berries and fruits. ${ }^{1}$ Pterostilbene, has numerous pharmacological properties that are similar to that of Resveratrol..$^{2-4}$ Pterostilbene has a better pharmacokinetic profile than resveratrol ${ }^{5}$ with a $\log \mathrm{P}$ value of 3.8. Among other pharmacological effects, Pterostilbene is also known to have anti-cancer potential. ${ }^{6-8}$ However, its poor aqueous solubility and stability posed a real challenge that needed to be circumvented. One promising strategy has been to deliver Pterostilbene ${ }^{9}$ using various nanoparticle-based drug vehicles including liposomes. Liposomes are spherical, micelle-like nano-carriers composed of phospholipids. ${ }^{10,11}$ Liposomes have the potential to encapsulate a large number of hydrophobic as well as hydrophilic drugs.
Submission Date: 12-11-2019; Revision Date: 27-02-2020; Accepted Date: 30-04-2020

DOI: 10.5530/ijper.54.2s.74 Correspondence: Dr.Suresh Palamadai Krishnan

Department of Biomedical Sciences, School of Biosciences and Technology, VIT, Vellore-632014, Tamil Nadu, INDIA.

Phone: +919444047684

E-mail: p.k.suresh@vit.ac.in

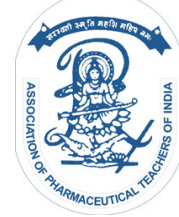

www.ijper.org 
Apart from being biocompatible, liposomal-drug formulation is studied for its site-specificity and low toxicity. This nano-formulation also protects the drugs from being degraded in vivo and increases the efficacy and bioavailability of the drugs. Therefore, various liposomal formulations are making their way to the market. ${ }^{12}$ DOTAP (1,2-dioleoyl-3-trimethylammoniumpropane) is a cationic surfactant, used in the formulation of liposomes. Cholesterol is a key component of cell membranes, which can be used in liposome formulation as a helper-lipid. Presence of cholesterol in liposomes is reported to provide stability to the liposomes and also can improve cellular uptake. ${ }^{13}$ Therefore, liposomes can efficiently encapsulate drugs and deliver it to the cells efficiently.

Our study involved the formulation of DOTAPCholesterol liposome-based delivery vehicle for Pterostilbene. Pterostilbene-encapsulated liposomes offer the possibility to entrap the drug and resolve the aforesaid solubility as well as stability-related issues, apart from possibly enabling better delivery to the target, thereby minimizing side effects. Specifically, DOTAP liposomes being cationic would be expected to target negatively charged chemical moieties on the cell membrane, necessitating site-specific drug administration for improved safety and efficacy. ${ }^{14}$ However, any variation in the net charge from positive to negative values may be dependent on the presence of cholesterol in the DOTAP liposome nano-construct. ${ }^{15}$ Hence, measurements of zeta potential (net charge) is an important determinant for nanoparticle behaviour in cell culture systems. Our work has reproducibly demonstrated the synthesis and characterization of Pterostilbene-encapsulated DOTAP liposomes. Our nano-constructs can be further studied to improve the ability in inducing cell death, possibly by an increase in its uptake/bioavailability.

\section{MATERIALS AND METHODS \\ Materials}

Pterostilbene, cholesterol and Amicon ${ }^{\circledR} 30 \mathrm{kDaUltracel}-\mathrm{PL}$ membrane ultra-centrifugal filters were obtained from Sigma-Aldrich, India. 1,2-dioleoyl-3-trimethylammoniumpropane (chloride salt) 18:1 TAP (DOTAP) was procured from Avanti Polar Lipids, Inc., USA. The remaining reagents used were of standard analytical grade.

\section{Liposome synthesis}

Pterostilbene-loaded liposomes as well as blank liposomes (unloaded) were prepared using the thin-film hydration method. ${ }^{15-18}$ Liposomes were composed of DOTAP and cholesterol (2:1, M ratios). Stock solutions of DOTAP and cholesterol as well as Pterostilbene were prepared in a solution containing chloroform: methanol in the ratio of 2:1 (v/v). For Pterostilbene-encapsulated liposomes, the drug: lipid ratio was kept the same at 0.1 (w/w). Blank liposomes were also synthesized with the same stoichiometry as described, minus the drug. The lipiddrug solution produced by the aforesaid thin-film hydration method was bath-sonicated $(20 \mathrm{kHz})$ for $15 \mathrm{~min}$. Then, probe sonication was done at $25 \%$ amplitude with 5 second pulse and 1 sec. pause for another $2 \mathrm{~min}$. The samples were then passed through a $0.2-\mu \mathrm{m}$ filter to ensure sterility and were stored at $4^{\circ} \mathrm{C}$.

\section{Liposome characterization-size, PDI, zeta potential}

The samples were subjected to $15 \mathrm{~min}$. of bath sonication, followed by dilution with ultra-pure, sterile (Milli-Q) water. Samples were further centrifuged at 5000xg in Ultracel-PL membrane ultra-centrifugal filter (Amicon ${ }^{\circledR}$ $30 \mathrm{kDa}$ ) for $5 \mathrm{~min}$. The top phase comprised of liposomes was collected and was again dispersed in ultra-pure (Milli-Q), sterile water and was characterized for its size (Z-average), PDI and zeta potential using Malvern's Zetasizer Nano ZS (ZEN 3600). ${ }^{16,19}$

\section{Liposome morphology- TEM}

Morphology of the liposomes was assessed using TEM. The samples were subjected to $15 \mathrm{~min}$. of bath sonication, followed by dilution with ultra-pure, sterile (Milli-Q) water. Samples were centrifuged at 5000xg in Ultracel-PL membrane ultra-centrifugal filter (Amicon ${ }^{\circledR} 30 \mathrm{kDa}$ ) for $5 \mathrm{~min}$. The top phase comprised of liposomes was collected and was again dispersed in sterile, ultra-pure (Milli-Q) water. An FEI-Tecnai G2 20S-TWIN highresolution TEM was used to analyse the samples. ${ }^{16,18}$

\section{Drug-lipid interactions in liposomes}

In order to study whether Pterostilbene interacts with DOTAP and cholesterol during and after liposome formation, we performed qualitative tests using Shimadzu UV-1280 UV-Vis spectrophotometer. Individual UV-Vis scans of DOTAP, cholesterol and Pterostilbene, dissolved in the same solvent that was used for the liposome synthesis (chloroform:methanol $(2: 1 \mathrm{v} / \mathrm{v})$ ), were recorded from $800 \mathrm{~nm}$ to $190 \mathrm{~nm}$. The liposome samples were subjected to $15 \mathrm{~min}$. bath sonication, followed by centrifugal filtration, so as to separate the free and encapsulated Pterostilbene. Individual UV-Vis scans of the top and the bottom phase of DOTAPcholesterol blank as well as Pterostilbene-loaded DOTAP-cholesterol liposomes were recorded from $300 \mathrm{~nm}$ to $190 \mathrm{~nm}$. Finally, the top phase of 
Pterostilbene-loaded DOTAP-cholesterol liposomes was lysed by diluting it 20-times with methanol followed by probe sonication at 25\% amplitude and $5 \mathrm{sec}$ pulse for 30 min. ${ }^{20} \mathrm{UV}-\mathrm{V}$ is scan of this lysate was also recorded from $800 \mathrm{~nm}$ to $190 \mathrm{~nm}$.

\section{Liposome Encapsulation efficiency}

The liposome samples (DOTAP-cholesterol blank and Pterostilbene-loaded liposomes) were subjected to $15 \mathrm{~min}$. of bath sonication. Free Pterostilbene was separated from the Pterostilbene-loaded liposomes by centrifuging it at $5000 \mathrm{xg}$ for $5 \mathrm{~min}$. at $4^{\circ} \mathrm{C}$ using Ultracel-PL membrane ultra-centrifugal filter (Amicon ${ }^{\circledR}$ $30 \mathrm{kDa}$ ). Free Pterostilbene was obtained at the bottom and the Pterostilbene-loaded liposomes were obtained at the top. In order to lyse the liposomes and measure the amount of encapsulated drug, the top phase was diluted 20-times with methanol, followed by $30 \mathrm{~min}$. of probe sonication at $25 \%$ amplitude and $5 \mathrm{sec}$. pulse. The bottom layer was also diluted with methanol and was mixed thoroughly. Standard graph of Pterostilbene in methanol was plotted $(318 \mathrm{~nm})$ and the top and bottom phases were read using Shimadzu UV-1280 UV-Vis spectrophotometer at $318 \mathrm{~nm} .{ }^{16,20}$

$$
\begin{aligned}
& \text { Encapsulation Efficiency }(\%)=\left(\text { Pterostilbene }_{(\mathrm{Top})} /\right. \\
& \text { Pterostilbene } \left._{(\text {(Top) }}+\text { Pterostilbene }_{(\text {Bottom })}\right) \text { x100 }
\end{aligned}
$$

Pterostilbene $_{\text {(тор) }}$ is the amount of Pterostilbene in the top phase, Pterostilbene ${ }_{(B o t t o m)}$ is the amount of Pterostilbene in the bottom phase.

\section{RESULTS}

\section{Liposome characterization-size, PDI, zeta potential}

The reproducibility of our measurements was demonstrated based on their size, PDI and zeta potential. The size of DOTAP-Cholesterol blank liposome was $415.1 \pm 41 \mathrm{~nm}(n=3)$, PDI was $0.477 \pm 0.02(n=3)$ and the zeta potential was $-21.6 \pm 1.4 \mathrm{mV}(n=3)$. The size of DOTAP-Cholesterol Pterostilbene-loaded liposome was little more than the blank liposome and was $435.6 \pm 50 \mathrm{~nm}(n=3)$. The PDI was $0.506 \pm 0.07 \quad(n=3)$, whereas the zeta potential was $-16.4 \pm 0.5 \mathrm{mV}(n=3)$.

\section{Liposome morphology- TEM}

The morphology of the liposomes was observed using TEM. Both, DOTAP-Cholesterol blank liposomes (Figure 1 (A)) as well as DOTAP-Cholesterol Pterostilbene-loaded liposomes (Figure 1 (B)) were circular in shape. Pterostilbene-loaded liposomes were slightly larger in size.

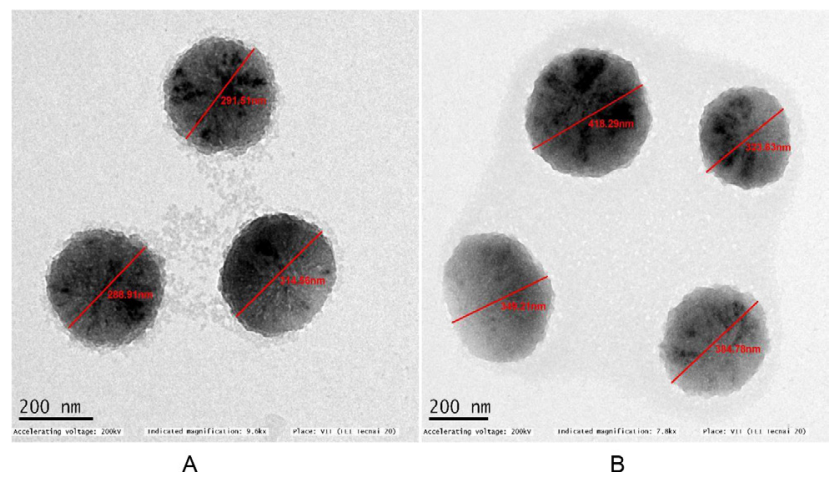

Figure 1(A): DOTAP-Cholesterol blank liposomes. Figure 1(B): Pterostilbene loaded DOTAP-Cholesterol liposomes.

TEM images reviled the circular morphology of liposomes. DOTAP-Cholesterol blank liposomes were smaller in size than Pterostilbene-loaded DOTAP-Cholesterol liposomes.

\section{Drug-lipid interactions in liposomes}

In order to study if there is any interaction between the liposome components, namely DOTAP, Cholesterol and Pterostilbene, UV-Vis spectrum scans were carried out for individual components as well as during and after liposome synthesis. UV-Vis $\lambda_{\max }$ for DOTAP was $238.5 \mathrm{~nm}$ (Figure 2 (A)), for that of cholesterol was $234 \mathrm{~nm}$ (Figure 2 (B)), whereas, when DOTAP and cholesterol were combined in 2:1 (M ratios), the $\lambda_{\text {max }}$ was found to be 239nm (Figure $2(\mathrm{C})$ ). The UV-Vis $\lambda_{\text {max }}$ for Pterostilbene was $318 \mathrm{~nm}$ (Figure 2 (D)). When DOTAP-cholesterol and Pterostilbene were combined during liposome synthesis, it gave two discrete lipid and Pterostilbene UV-Vis $\lambda_{\max }$ peaks at $239.5 \mathrm{~nm}$ and $319 \mathrm{~nm}$ (Figure $2(\mathrm{E})$ ) respectively. After centrifugation using the AMICON filter, the top phase contained the liposomes, while the bottom phase contained buffer and free drug (if any). The UV-Vis spectrum of the bottom phase of DOTAP-cholesterol blank liposome gave no peak (Figure $2(\mathrm{~F})$ ), while the UV-Vis spectrum of the top phase of DOTAP-cholesterol blank liposome gave a peak at $217 \mathrm{~nm}$ (Figure $2(\mathrm{G})$ ), thus suggesting that the top phase was comprised of the liposomes and the bottom phase only had the buffer. The UV-Vis spectrum of the bottom phase of DOTAP-cholesterol Pterostilbene-loaded liposome gave no major peak (Figure $2(\mathrm{H})$ ) while the UV-Vis spectrum of the top phase of DOTAP-cholesterol Pterostilbene-loaded liposome gave a peak at 214nm (Figure 2 (I)), but when the DOTAP-cholesterol Pterostilbene-loaded liposome top phase was subjected to probe sonication as described above, it gave UV-Vis $\lambda_{\max }$ at $221 \mathrm{~nm}$ and at $318.5 \mathrm{~nm}$ (Figure $2(\mathrm{~J})$ ). This indicates that the top phase contains the liposomes and when the liposomes are subjected to probe sonication, the liposomes are 

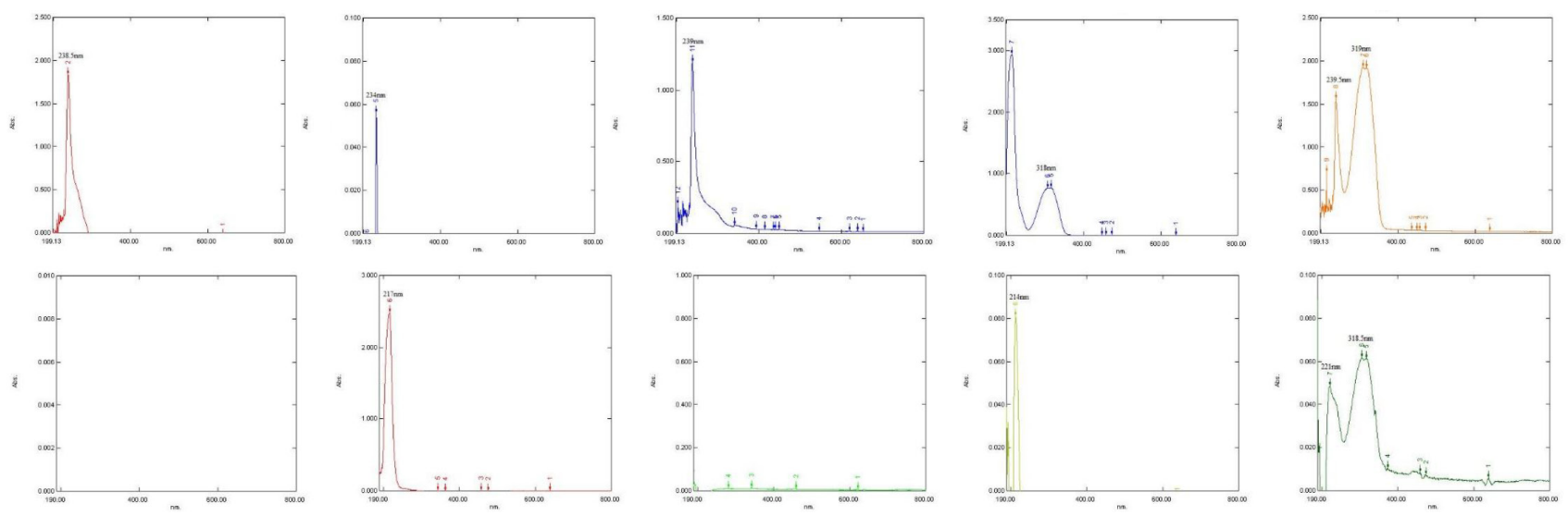

Figure 2: These figures represent UV-Vis spectrophotometer scans of the liposome components viz., DOTAP, Cholesterol as well as of Pterostilbene before liposome preparation, during liposome preparation as well as after liposome preparation as mentioned in the results. Figure 2(A). DOTAP, Figure 2 (B). Cholesterol, Figure 2 (C). DOTAP and cholesterol combined in 2:1 (M ratios), Figure 2 (D). Pterostilbene, Figure2 (E). DOTAP-cholesterol and Pterostilbene in combination, Figure 2 (F). Bottom phase of DOTAP-cholesterol blank liposome, Figure2 (G). Top phase of DOTAP-cholesterol blank liposome, Figure 2 (H). Bottom phase of DOTAP-cholesterol Pterostilbene-loaded liposome, Figure 2 (I). Top phase of DOTAP-cholesterol Pterostilbene-loaded liposome, Figure 2 (J). DOTAP-cholesterol Pterostilbene-loaded liposome top phase after probe sonication.

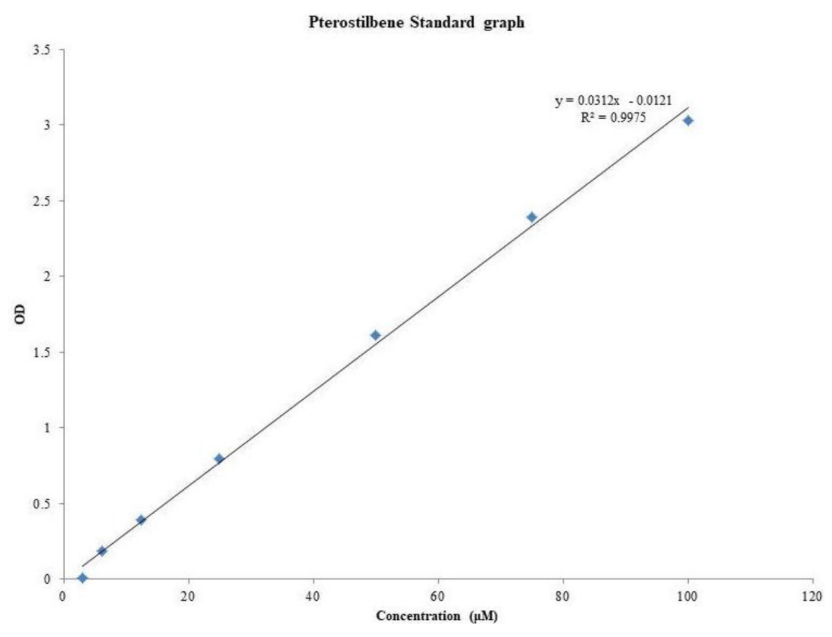

Figure 3: Standard graph of Pterostilbene was plotted at $318 \mathrm{~nm}$. Solvent used was methanol.

lysed and Pterostilbene escapes out. These results show that there is no interaction in the UV-Vis absorbance of various components of the liposomes, viz. DOTAP, Cholesterol and Pterostilbene. The data also revealed that Pterostilbene-loaded DOTAP-Cholesterol liposomes did not display the Pterostilbene UV-Vis absorbance peak, unless subjected to lysis, indicating that Pterostilbene is encapsulated inside the liposomes.

\section{Liposome Encapsulation efficiency}

UV-Vis Spectrophotometer was used to determine the encapsulation efficiency of the liposomes. The Pterostilbene content in the top and the bottom phase was quantified by comparing the OD with the standard graph of Pterostilbene. OD of the bottom phase was 0.023 and the concentration of Pterostilbene was $1.12 \mu \mathrm{M}$, while the OD of the top phase after probe sonication was 1.4 which corresponded to $45.25 \mu \mathrm{M}$ Pterostilbene. The standard graph of Pterostilbene is showed in Figure 3. The encapsulation efficiency was reported to be $97.5 \pm 0.8 \%(n=3)$.

\section{DISCUSSION}

Pterostilbene has been studied earlier for its various properties, including action against various types of cancers such as human gastric carcinoma, leukemia and human colon cancer. Due to its higher $\log P$ value ( $\log$ P 3.8), Pterostilbene is not soluble in aqueous media, which can hinder its delivery in vitro and in vivo. Also, Pterostilbene uptake in Estrogen Receptor-negative cells is a challenge. One of the key pharmacokinetic advantages of Liposome-entrapment of a drug can be possible decrease in the dose required for the therapeutic effect. Further, liposome encapsulation circumvents solubility-related issues of lipid-soluble drugs that will serve to improve the dispersion of our nano-construct in a suitable vehicle as well as contribute to an enhancement in the uptake. Blank and loaded liposomes were synthesized and Pterostilbene was encapsulated within the loaded liposomes. They were characterized for their shape, size, PDI and zeta potential using DLS and TEM. The size observed in the TEM images was fairly similar to that obtained from the DLS data. The average size of ours is similar, barring a slight variation, to the values reported in the literature with the unextruded liposomes being relatively larger. ${ }^{21,22}$ Polydispersity of both 


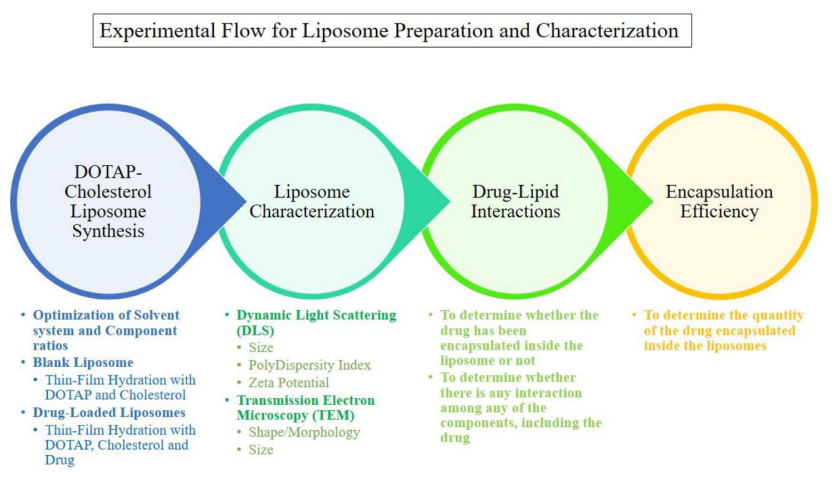

Figure 4: Experimental flow that can be used by educators involved in drug delivery.

the liposomes suggested a uniform size distribution. ${ }^{16,23}$ The zeta potential, however, was negative, despite the presence of DOTAP, a cationic lipid. One possibility of this cationic lipid acquiring a negative charge might be due to the presence of Cholesterol, which is consistent with certain reports in the literature. ${ }^{15}$ There might also be a formation of a protein adsorption layer or protein corona around the liposomes, which can modulate the uptake mechanism ${ }^{16,24}$ thereby circumventing the negative charge-mediated barriers for the uptake processes. ${ }^{25,26}$ Taken together, our results conclusively demonstrate that Pterostilbene can be encaspsulated in DOTAP-liposomes fairly reproducibly. This synthesis and characterization has provided a basis for their testing in cell culture systems for possible improvements in cytotoxicity and cell death potential. Also, this work can be extended in model systems in vivo as well.

\section{CONCLUSION}

This work reports the hitherto undocumented synthesis and preliminary characterization of Pterostilbeneencapsulated DOTAP liposomes. This approach would pave the way for evaluating the in vitro and in vivo cancer cell death potential of Pterostilbene in the DOTAP liposome-based delivery vehicle. Validation of our experimental flow has paved the way to encapsulate other molecules in the stilbenoid class, followed by their subsequent characterization. Also, this design can be an iterative experimental tool (Figure 4 -experimental flow-chart for the educators) for the demonstration of the encapsulation, characterization and subsequent enhancements in bioavailability by pharmaceutical educators as well.

\section{Authors' Contributions}

The lead author was primarily responsible for executing the project in terms of standardization, trouble-shooting and performing experiments (at the bench). He was also responsible for writing the first draft of the manuscript. The second and corresponding author was involved with the conceptualization, data interpretation, trouble-shooting as well as editing and critical evaluation of the manuscript. He is the mentor of the first author of this manuscript.

\section{ACKNOWLEDGEMENT}

The authors thank VIT for their constant encouragement and support as well as for their unlimited WIFI connectivity. The corresponding author thanks DST (SERB) for funding a project on drug delivery systems involving Luteolin ((SB/SO/HS-157 (2013)). Last but not least, the corresponding author also thanks the other research scholars as well as the short-term project students for their support.

\section{CONFLICT OF INTEREST}

The authors declare no conflict of interests.

\section{ABBREVIATIONS}

DOTAP: 1,2-dioleoyl-3-trimethylammonium-propane; TEM: Transmission Electron Microscopy; kHz: Kilo Hertz; PDI: Polydispersity Index; DLS: Dynamic Light Scattering; EE: Encapsulation Efficiency; kDa: Kilo Dalton; UV-Vis: Ultraviolet -Visible; OD: Optical Density.

\section{REFERENCES}

1. Siedlecka-Kroplewska K, Jozwik A, Kaszubowska L, Kowalczyk A, Boguslawski W. Pterostilbene Induces Cell Cycle Arrest and Apoptosis in MOLT4 Human Leukemia Cells. Folia Histochemica Cyotbiologica. 2012;50(4):574-80.

2. Perečko T, Drábiková K, Račková L, Číž M, Podborská M, Lojeka A, et al. Molecular Targets of the Natural Antioxidant Pterostilbene: Effect on Protein Kinase C, Caspase-3 and Apoptosis in Human Neutrophils in vitro. Neuro Endocrinol Lett. 2010;31(Suppl 2):84-90.

3. Pan MH, Chang Y, Badmaev V, Nagabhushanam K, Ho C. Pterostilbene Induces Apoptosis and Cell Cycle Arrest in Human Gastric Carcinoma Cells. J Agric Food Chem. 2007;55(19):7777-85.

4. Sirerol JA, Feddi F, Mena S, Rodriguez ML, Sirera P, Aupí M, et al. Free Radical Biology and Medicine Topical Treatment with Pterostilbene, a Natural Phytoalexin, Effectively Protects Hairless Mice against UVB RadiationInduced Skin Damage and Carcinogenesis. Free Radical Biology and Medicine. 2015;85:1-11.

5. Sirerol JA, Feddi F, Mena S, Rodriguez ML, Sirera P, Aupí M, et al. Free Radical Biology and Medicine Topical Treatment with Pterostilbene, a Natural Phytoalexin, Effectively Protects Hairless Mice against UVB RadiationInduced Skin Damage and Carcinogenesis. Free Radical Biology and Medicine. 2015;85:1-11.

6. Dellinger RW, Garcia AM, Meyskens FL. Differences in the Glucuronidation of Resveratrol and Pterostilbene: Altered Enzyme Specificity and Potential Gender Differences. Drug Metab. Pharmacokinet. 2014;29(2):112-9.

7. Schneider JG, Alosi JA, Mcdonald DE, Mcfadden DW. Pterostilbene Inhibits Lung Cancer Through Induction of Apoptosis 1. Journal of Surgical Research. 2010;161(1):18-22. 
8. Wang Y, Shen Y, Xu J, Han K, Zhou Y, Yang S, et al. Pterostilbene Suppresses Human Endometrial Cancer Cells in vitro by down-Regulating miR-663b. Nature Publishing Group. 2017;38(10):1394-400.

9. McCormack D, Mcfadden D. Pterostilbene and Cancer: Current Review. Journal of Surgical Research. 2012;173(2):e53-61.

10. Zhang Y, Shang Z, Gao C, Du M, Xu S, Song H, et al. Nanoemulsion for Solubilization, Stabilization and in vitro Release of Pterostilbene for Oral Delivery. AAPS Pharm Sci Tech. 2014;15(4):1000-8.

11. Puri A, Loomis K, Smith B, Lee J, Yavlovich A, Heldman E, Blumenthal R. Lipid-Based Nanoparticles as Pharmaceutical Drug Carriers: From Concepts to Clinic. Critical Reviews in Therapeutic Drug Carrier Systems. 2009;26(6):523-80.

12. Zhong $\mathrm{Y}$, Wang J, Wang $\mathrm{Y}, \mathrm{Wu}$ B. Preparation and Evaluation of LiposomeEncapsulated Codrug LMX. International Journal of Pharmaceutics. 2012;438(1-2):240-8.

13. Akbarzadeh A, Rezaei-Sadabady R, Davaran S, Woo JS, Zarghami N, Hanifehpour $\mathrm{Y}$, et al. Liposome: Classification, preparation and applications. Nanoscale Research Letters. 2013;8(1):102.

14. Crook K, Stevenson BJ, Dubouchet M, Porteous DJ. Inclusion of cholesterol in DOTAP transfection complexes increases the delivery of DNA to cells in vitro in the presence of serum. Gene Therapy. 1998;5(1):137-43.

15. Sercombe L, Veerati T, Moheimani F, Wu Y, Sood AK, Hua S. Advances and Challenges of Liposome Assisted Drug Delivery. Frontiers in Pharmacology. 2015;6:1-13.

16. Moon D, Mccormack D, Mcdonald D, Mcfadden D. Pterostilbene induces mitochondrially derived apoptosis in breast cancer cells in vitro. Journal of Surgical Research. 2013;180(2):208-15.

17. Sinha A, Suresh PK. Enhanced Induction of Apoptosis in HaCaT Cells by Luteolin Encapsulated in PEGylated Liposomes: Role of Caspase-3. Appl Biochem Biotechnol. 2018;188(1):147-64.

18. Johnston MJW, Edwards K, Karlsson G, Cullis PR. Influence of Drugto-Lipid Ratio on Drug Release Properties and Liposome Integrity in
Liposomal Doxorubicin Formulations. Journal of Liposome Research. 2008;18(2):145-57.

19. Liu X, Ruan L, Mao W, Wang J, Shen Y, Sui M. Preparation of RGDModified Long Circulating Liposome Loading Matrine and Its in vitro. Anti-Cancer Effects. International Journal of Medical Sciences. 2010;7(4):197-208.

20. Choksi A, Sarojini KVL, Vadnal P, Dias C, Suresh PK, Khandare J. Comparative Anti-Inflammatory Activity of Poly (Amidoamine) (PAMAM) Dendrimer - Dexamethasone Conjugates with Dexamethasone-Liposomes. International Journal of Pharmaceutics. 2013;449(1-2):28-36.

21. Ai X, Zhong L, Niu H, He Z. Thin-Film Hydration Preparation Method and Stability Test of DOX-Loaded Disulfide-Linked Polyethylene Glycol 5000-Lysine-Di-Tocopherol Succinate Nanomicelles. Asian Journal of Pharmaceutical Sciences. 2014;9(5):244-50.

22. Wiethoff CM, Smith JG, Koe GS, Middaugh CR. The potential role of proteoglycans in cationic lipid-mediated gene delivery. Studies of the interaction of cationic lipid-DNA complexes with model glycosaminoglycans. J Biol Chem. 2001;276(35):32806-13.

23. Sudhakar B, Varma JNR, Murthy KVR. Formulation, Characterization and ex vivo Studies of Terbinafine $\mathrm{HCl}$ Liposomes for Cutaneous Delivery. Current Drug Delivery. 2014;11(4):521-30.

24. Seleci M, Seleci DA, Scheper T, Stahl F. Theranostic LiposomeNanoparticle Hybrids for Drug Delivery and Bioimaging. Int J Mol Sci. 2017;18(7):1415.

25. Palchetti S, Colapicchioni V, Digiacomo L, Caracciolo G, Pozzi D, Capriotti AL, et al. The protein corona of circulating PEGylated liposomes. Biochimica et Biophysica Acta. 2016;1858(2):189-96.

\section{PICTORIAL ABSTRACT}

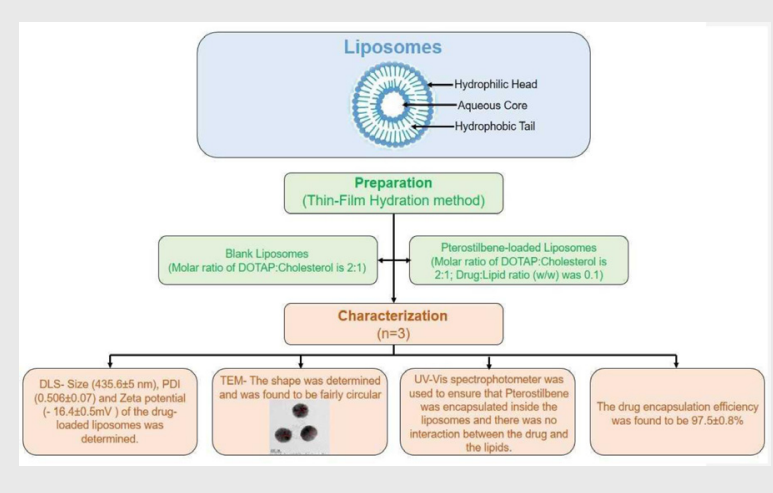

\section{SUMMARY}

We have demonstrated reproducibly the synthesis and characterization of Blank \& Pterostilbene-encapsulated DOTAP-cholesterol liposomes. Data obtained was in consonance with published data.

\section{About Authors}

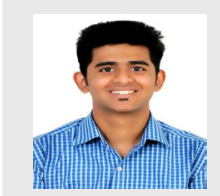

Hiray K.S: He has finished his Bachelor of Technology in Biotechnology from VIT Vellore. He is currently pursuing his Master of Technology by Research and is working on the effects of drugs (purified natural compounds) on cancer cells and formulation of an effective nano-derived drug delivery system. 
P.K.Suresh: Professor Higher Academic Grade (PHAG) in the Department of Biomedical Sciences, School of Biosciences and Technology. He has approximately 21 years of teaching, research and administrative experience (post-Ph.D.). He received his second masters and Ph.D. in SIUE, IL, USA and the University of Cincinnati, Ohio, USA respectively. He was a Postdoctoral fellow at the University of Texas at Austin, TX, USA as well as Rutgers University, Piscataway, USA. P.K.Suresh has authored/co-authored over 45 publications in SCOPUS-indexed journals with an $b$-index of 10 and a cumulative citation index of 407 . He has been a resource person and/or coordinator in several Faculty Development Programs as well as in International Conferences in India and overseas. He has mentored students at several levels including those pursuing their doctoral degree. Apart from in silico and in vitro Chemical Biology/Technology, he is also involved in drug development and delivery systems.

Cite this article: Hiray KS, Krishnan SP. Synthesis and Characterization of Un-encapsulated and Pterostilbeneencapsulated DOTAP: Cholesterol Liposomes. Indian J of Pharmaceutical Education and Research. 2020;54(2s):s182-s188. 